\title{
STIR, SPIR and SPAIR techniques in magnetic resonance of the breast: A comparative study
}

\author{
M. Margarida Ribeiro ${ }^{1,2 *}$, Liliana Rumor ${ }^{1,3}$, Marta Oliveira ${ }^{1,3}$, J. Goiry O’Neill, ${ }^{2,4}$, Cruz Maurício ${ }^{2,5}$ \\ ${ }^{1}$ Department of Sciences and Radiation Technologies, Scientific Area of Radiology from Polytechnic Institute, Lisbon, Portugal \\ ${ }^{2}$ Anatomy Department, Medical Sciences Faculty of Lisbon, Lisbon, Portugal \\ ${ }^{3}$ Faculty of Medicine in Pilsen, Charles University, Prague, Czech Republic \\ ${ }^{4}$ Department of Physics, Centre of Physics and Technological Research, Sciences and Technology Faculty, Caparica, Portugal \\ ${ }^{5}$ EUROMEDIC-Medical Imaging, Tomar, Portugal \\ Email: * margarida.ribeiro@estesl.ipl.pt
}

Received 15 January 2013; revised 18 February 2013; accepted 25 February 2013

\section{ABSTRACT}

The amount of fat is a component that complicates the clinical evaluation and the differential diagnostic between benign and malign lesions in the breast MRI examinations. To overcome this problem, an effective erasing of the fat signal over the images acquisition process, is essentials. This study aims to compare three fat suppression techniques (STIR, SPIR, SPAIR) in the MR images of the breast and to evaluate the best image quality regarding its clinical usefulness. To mimic breast women, a breast phantom was constructed. First the exterior contour and, in second time, its content which was selected based on 7 samples with different components. Finally it was undergone to a MRI breast protocol with the three different fat saturation techniques. The examinations were performed on a 1.5 T MRI system (Philips ${ }^{\circledR}$ ). A group of 5 experts evaluated 9 sequences, 3 of each with fat suppression techniques, in which the frequency offset and TI (Inversion Time) were the variables changed. This qualitative image analysis was performed according 4 parameters (saturation uniformity, saturation efficacy, detail of the anatomical structures and differentiation between the fibroglandular and adipose tissue), using a five-point Likert scale. The statistics analysis showed that anyone of the fat suppression techniques demonstrated significant differences compared to the others with $(p>0.05)$ and regarding each parameter independently. By Fleiss' kappa coefficient there was a good agreement among observers $P(e)=0.68$. When comparing STIR, SPIR and SPAIR techniques it was confirmed that all of them have advantages in the study of the breast MRI. For the studied parameters, the results through the Friedman Test showed that there are similar advantages applying anyone of these techniques.

\footnotetext{
${ }^{k^{*}}$ Corresponding author
}

Keywords: Fat Tissue; Magnetic Resonance; Breast MRI; Fat Suppression

\section{INTRODUCTION}

Magnetic Resonance Imaging (MRI) combines some interesting principles of physics and some sophisticated technology to make medical images of amazing clarity and surprising high diagnostic accuracy. MRI today is more revolutionary than $\mathrm{x}$-ray imaging was in a century ago. Twenty-five years ago, when MRI was first introduced in the clinical practice, its prosperity of application to medical imaging could not have been imagined. Its quickly development was demonstrated and MRI is useful in the diagnosis of diseases [1]. The rise of MRI is continuing to unfold itself. Breast imaging is one more example of the versatility of MRI [1,2].

In 1986, MRI was used for the first time in the study of the breast and, this diagnostic method has undergone significant advances, revealing that it is promising in the investigation of breast cancer.

MRI of the breast is a non-invasive technique with high tissue differentiation. This technique has been widely discussed and evaluated, mainly because it provides additional data not possible to obtain by conventional imaging methods, such as mammograms or ultrasound. Breast MRI is particularly useful in the evaluation of newly diagnosed breast cancer, in women whose breast tissue is very dense, and not suitable for screening in women with a high lifetime risk of breast cancer due to their family history or genetic disposition [1,3].

Due the high sensitivity of MRI, there are high falsepositive rates, leading to a large number of unnecessary biopsies. Thus, the MRI shows itself to be one technique of high sensitivity, but with low specificity (promotes sensitivities above 95\% and specificities no more than $80 \%$ for breast tumours of all sizes) [4]. To optimize the quality of the morphologic and kinetic information 
yielded by breast MR imaging, the radiologist must attend to several practical, technical and clinical inclusion criteria, such as the fat suppression, which is used in routine magnetic resonance imaging for many purposes [5-8].

This study aims to compare three fat suppression techniques (STIR, SPIR, SPAIR) in the MR images of the breast and to evaluate the best image quality regarding its clinical usefulness.

We think to be possible to give a contribution in the optimization of fat suppression techniques in breast MRI examinations and to provide information to the decision of the best fat suppression technique have to should apply. This theme is important in order to increase the pathological evidences and the differentiation between the breast tissues.

The specific aim to carry out the method was to construct a breast phantom able to reproduce a good quality breast MRI to determine which sequence would be more advantageous.

\section{Fat Suppression Techniques}

Fat suppression is commonly used in MRI to suppress the signal from adipose tissue and to enhance the evidence of the abnormalities inside the adipose tissue. The signal suppression of the fat can be achieved through three different methods: 1 -applying a pulse of the fat saturation time adjusted, 2-by inversion-recovery or 3-imaging and opposed-phase imaging [9].

However, we will discuss here the fat saturation pulse and the inversion-recovery imaging used to suppress the signal from normal adipose tissue, in order to increase image quality and reduce chemical shift artifact or improve the visualization of the breast. The optimal fat suppression technique depends on the amount of fat tissue that requires signal suppression. Fat suppression is a generic term that includes several techniques, each one with specific advantages, disadvantages and pitfalls [9, 10]. Lipid protons and hydrogen protons from water behave differently during an MRI acquisition and the success of the fat suppression techniques, within the other criteria, are based on those differences. Two major properties are involved: first, there is a small difference in the resonance frequency, between lipid and water protons, which is related to the different electronic environments [9]. This so-called chemical shift allows frequency-selective fat saturation. Second, the difference in T1 between adipose tissue and water can be used to suppress the fat signal with inversion-recovery techniques [11]. We will describe below the main features of the each one fat suppression techniques studied: STIR, SPIR and SPAIR.

The STIR sequence (Short TI Inversion Recovery) applies an inversion pulse and time delay (TI) into the range of the time inversion values from the lipomatous tissue (Figure 1). The aim is to null the longitudinal magnetization. So that fat will not contribute with signals in any subsequent image. It is an inversion-recovery pulse sequence with specific timing in order to suppress the signal from fat tissue. After the $180^{\circ}$ inversion pulse, the longitudinal magnetization of fat/adipose tissue will recover faster than that of water. The $180^{\circ}$ radiofrequency (RF) pulse is used to invert water and fat spins, and then waits about $180 \mathrm{~ms}$ at 1.5 Tesla for the more rapidly-recovering fat peak to reach the null point (i.e. the point at which it passes through the transverse plane). At this point, a $90^{\circ}$ "check" pulse flips the magnetization into the transverse plane so that the fat peak is zero but the water peak, which still had a negative $\mathrm{z}$ component, is present. When this $90^{\circ} \mathrm{RF}$ pulse is applied, there is no longitudinal magnetization to be flipped into the $x-y$ plane from fat [9]. The fat will reach its null point faster than water and other components and an image of these structures can be generated. Contrast between tissues depends entirely from the $\mathrm{T} 1$ differences at this stage. Tissues with a different $\mathrm{T} 1$ relaxation time relating to fat have a signal because they either have not yet reached the null point, or have recovered some relaxation past this point.

This method has several advantages: it suppresses the signal of adipose tissue, including the water fraction and it is the only method which more protects the images against the magnetic field in homogeneities.

The inversion-recovery technique can be modified by using chemical selective or spectral pre-saturation attenuated inversion-recovery pre-pulses.

SPIR sequence (Spectral Pre-saturation with Inversion-Recovery) is a combination of the spectral saturation and STIR routines. Spectral fat suppression involves an RF preparation pulse which, instead of rotating the magnetization completely, is carefully tuned to the frequency of fat only. This is possible because the different

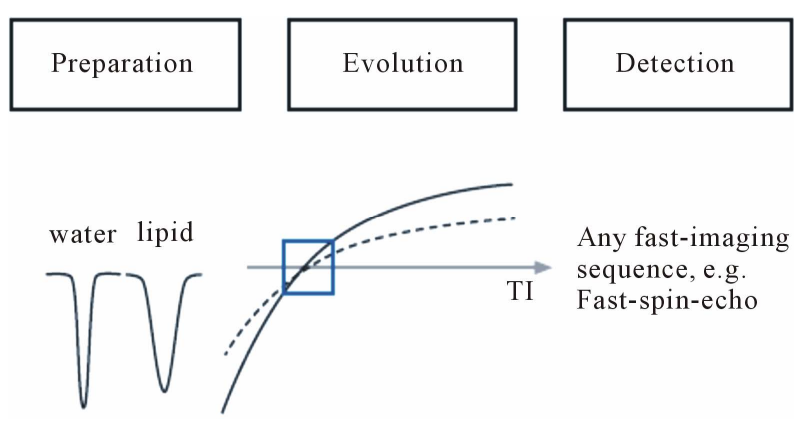

Figure 1. Diagram of STIR consists of three time periods, namely the preparation period (nonselective inversion), the evolution period (T1 recovery), and the detection period. Note that any other tissue (dashed line) with a T1 similar to that of lipids (solid line) is nulling likewise (source: Lee, Yiel, 2011 [23]). 
molecular environment of $1 \mathrm{H}$ proton magnetizations in fat-means they precess, at a slightly different frequency. In this technique, a RF pulse at the precessional frequency of the fat is applied to the imaging volume with a magnitude of $180^{\circ}$. The magnetic moments of fat are, therefore, totally inverted into the $\mathrm{z}$ direction. After a time TI, which corresponds to the null point of fat, the $90^{\circ}$ excitation pulse is applied. As fat has no longitudinal magnetization at this point, the excitation pulse produces no transverse magnetization to the fat. Therefore the fat signal is null and void in image [12].

It is a hybrid sequence obtained by the prior pulse of $\mathrm{RF}$, which will constrain the variation of the magnitude of the peak signal of fat [13].

This technique combines fat saturation and inverting mechanisms similar to the STIR to eliminate the fat signal. However, it has several advantages over STIR. The SPIR is much less susceptible to magnetic field inhomogeneities, because fat signal annulment also occurs by selecting an inversion time that corresponds to the null point of fat. This depends on the $\mathrm{T} 1$ recovery time of fat rather than its precessional frequency, and the relaxation times are not affected by small changes in homogeneity [9].

The fat suppression technique SPAIR (Spectral Adiabatic Inversion Recovery) is a powerful technique for fat suppression, which offers different advantages over conventional fat suppression techniques. The technique is characterized by a low sensitivity to RF field inhomogeneities and only fat spins are suppressed/inverted (Figure 2). SPAIR uses a spectral selective adiabatic inversion pulse to invert the fat spins in the analysed volume. After the adiabatic pulse, a large spoiler is used in order to destroy any remaining transverse magnetization $[9,14]$. The fat spins will now decay according to the T1 relaxation rate, and after a certain characteristic time (TI null) the longitudinal magnetization will be zero. At this point, an excitation pulse is applied. As the fat spins have zero longitudinal magnetization at this point, they will

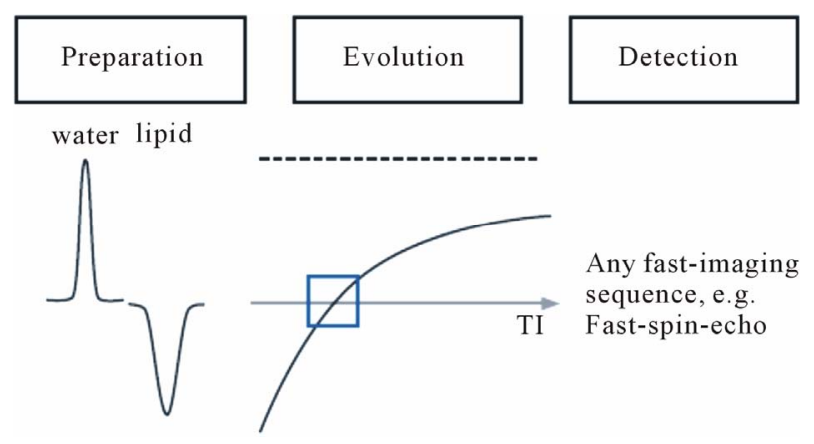

Figure 2. Diagram of SPAIR. The spectral selective inversion pre-pulse is set on the lipid frequency, leaving any other tissue unaffected (dashed line) during both the preparation and the evolution period (source: Lee, Yiel, 2011 [23]). not contribute to the MR signal.

The implementation of SPAIR fat suppression techniques will result in a more homogeneous fat saturation compared to previous fat suppression techniques.

\section{MATERIAL AND METHODS}

\subsection{Phantom Construction}

This study has characteristics that include the manipulation of variables and setting up experimental situations in the natural environment. The study follows the qualitative paradigm with weighting of response levels in order to be analyzed statistically.

The purpose was to evaluate the best image quality concerning the fat saturation strategies. The mediator variable is the fat suppression and the independent variables are the techniques STIR, SPIR and SPAIR.

MRI phantoms play an important role in the calibration and checking of MRI equipment, in the development of new systems, pulse sequences and in the training of MRI operators. Due the time consuming and the RF deposition be unknown at start point and regarding the ethical issues related with the use of volunteers, the authors decided to perform the experimental component with a phantom.

Two types of MRI phantoms are commonly used: those of an aqueous solution and those that are jelly-like as a material.

According to Kato et al., a gel carrageenan (CAGN) phantom is equivalent to most human tissues for electrical conductivity and relaxation times. The CAGN phantom is highly useful for experiments such as in MRI [15].

Carrageenan, unlike agarose, has little effect on T1 and $\mathrm{T} 2$ relaxation times.

CAGN is a linear sulphated polysaccharide, which is extracted from red seaweeds, and therefore very difficult to find available for use. For this reason, the carrageenan was replaced by another jelly-like agent-the agarosewhich was mixed with water and gelatine and heated in a microwave until it boiled. The mixture was then cooled to solidify it.

Based on the literature available, initially to select the final phantom content, some trials were made by mixing different ingredients [16,17] (Table 1). All these composition procedures and its amount were rigorously carried out in collaboration with the Chemistry laboratory of Polytechnic Institute and its experts (Table 2).

These mixtures were introduced in sterilized packages (Figure 3) and submitted to a MRI examination to determine which one was more similar with the breast tissue. The MRI protocol acquisition was composed by:

1) Survey;

2) T2 weighted TSE in Coronal plan;

3) T2 weighted TSE in Coronal plan with SPIR appli- 
Table 1. Description and composition of phantom samples that composed the seven samples shown in the Figure 3.

\begin{tabular}{cc}
\hline Samples & Ingredients \\
\hline 1 & Agarose; gelatine \\
2 & Agarose; gelatine; gadolinium chloride (GdCl3) \\
3 & Agarose; gelatine; GdCl3; olive oil \\
4 & Agarose; gelatine; GdCl3; corn oil \\
5 & Agarose; gelatine; GdCl3; pork fat \\
6 & Turkey breast; pork fat (small geometry) \\
7 & Turkey breast; pork fat (large geometry) \\
\hline
\end{tabular}

Table 2. Ingredients and their quantity in the breast phantom composition.

\begin{tabular}{|c|c|c|}
\hline Ingredients & Composition (100 g) & Weight \\
\hline Agarose & - & $17.35 \mathrm{~g}$ \\
\hline Gelatine & - & $29.7 \mathrm{~g}$ \\
\hline Distilled water & - & $990 \mathrm{ml}$ \\
\hline Turkey breast $\left(\mathrm{RB}^{*}\right)$ & $\begin{array}{l}\text { Protein-15.5 g } \\
\text { Lipids-0.8 g }\end{array}$ & $150 \mathrm{~g}$ \\
\hline Pork fat $\left(\mathrm{RB}^{*}\right)$ & $\begin{array}{l}\text { Saturated fats-38\% - } 43 \% \\
\text { Unsaturated fats- } 56 \%-62 \%\end{array}$ & $55.56 \mathrm{~g}$ \\
\hline Turkey breast (LB*) & $\begin{array}{l}\text { Protein—15.5 g } \\
\text { Lipids- } 0.8 \mathrm{~g}\end{array}$ & $120 \mathrm{~g}$ \\
\hline Pork fat $\left(\mathrm{LB}^{*}\right)$ & $\begin{array}{l}\text { Saturated fats-38\% - } 43 \% \\
\text { Unsaturated fats- } 56 \%-62 \%\end{array}$ & $66.67 \mathrm{~g}$ \\
\hline
\end{tabular}

"RB—right breast; ${ }^{*} \mathrm{LB}$-left breast.

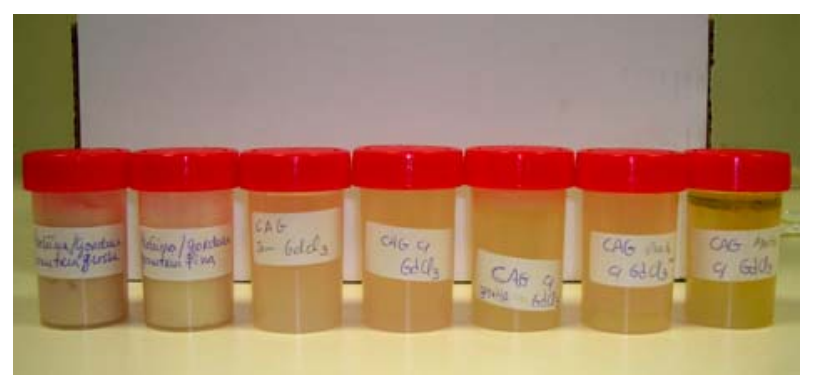

Figure 3. Samples within sterilized bottles. The content is from the left (sample 1) to right (sample 7) the composition described in the Table 1.

ance (Frequency offset: $100 \mathrm{~Hz}$ );

4) T2 weighted TSE in Coronal plan SPAIR (Inversion delay: $120 \mathrm{~ms})$;

5) $\mathrm{STIR}(\mathrm{TI}=90 \mathrm{~ms}$; TI = $180 \mathrm{~ms}$; TI = $135 \mathrm{~ms}$ );

6) T1 weighted TSE in Coronal plan.

The mature female breast is composed mainly by four structures: lobules or breast glands, lactiferous ducts, fat and connective tissue. The lobules group together form larger units called lobes. In the distribution of the lobes there is a preponderance of glandular tissue in the upper outer portion of the breast. At the surround of the lobes there is breast fat. The breasts of younger women are primarily composed of glandular tissue, with only a small percentage of the fat. With woman age and with the loss of estrogens at menopause, the glandular tissue in the lobes regresses and is replaced progressively by fat $[18,19]$.

After the evaluation of the sample's images it was selected the most consistent with the proposed target, i.e., its similarity with breast anatomy. Thus, as for phantom content the sample chosen was the seventh sample. In this study, the agarose and the gelatine (gelling agents) were used to provide the phantom with sufficient strength and long enough $\mathrm{T} 1$ and $\mathrm{T} 2$ values to mimic the human tissue [14].

In order to simulate the heterogeneity of fat and glandular tissue that occurs within breasts, the pork fat representing the breast adipose tissue and the turkey breast representing the breast parenchyma were added in different quantities on each breast. The left breast had $20 \%$ less of turkey breast and $20 \%$ more of pork fat than the right breast [16] (Figure 4).

Cubes of turkey breast were used in equal size, mixed with pork fat by means of a glass rod. The volume of turkey breast added to the phantom was determined by water displacement, while the volume for liquefied pork fat was measured. So that the phantom looks similar to the anatomical region studied, a mould of traditional plaster Biplastrix ${ }^{\circledR}$ was designed, which was rubberized with a polyethylene bag and its inside coated with the Sample 1, and then filled with the Sample 7 (large geometry), according to Table 1.

The mould in which the final composition of the study was introduced was built based on a human model in the Prosthesis laboratories, so it mimics, as best as possible, the breasts of a woman with morphometric characteristics measured by a bra with D cup and $35 \mathrm{~cm}$ width (Figure 5).

The phantom ingredients were used in the proportions shown in Table 2.

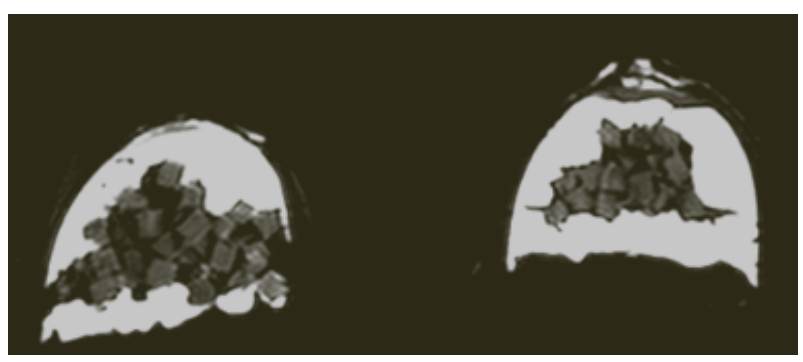

Figure 4. MR image of the breast phantom. We can see at the center the turkey breast represented by the fibroglandular tissue and, in the periphery, the fat tissue represented by pork fat and jelly agents. 




Figure 5. MRI breast phantom positioned inside the coil is a sensitive surface coil with a place to dovetail both breasts. The coil goes into the tunnel with the woman in ventral position. The prototype only differs on the weight and volume of the women trunk.

Its effectiveness, before use, was evaluated in two phases: 1) was submitted to a three sequences of fat suppression, STIR, SPIR and SPAIR. The signal intensity, trough square ROI(s), was measured in breast fat tissue of the phantom. Its similarity with the human breast was proved by the value of the signal intensity of the breast tissues as a young breast on the left side and a mature breast (middle age pattern) on the right side. Placed in the retro-glandular tissue exactly behind the pouch serous of Chassaignac, the average of the signal intensity was to the STIR sequence (559.192 \pm 40.21 ), to the SPIR was $(410.14 \pm 53.58)$ and to the SPAIR was (508.23 \pm 35.02). The ROI(s) had less than $50 \mathrm{~mm}^{2}$ in all measures. The same values obtained in the human breast were in a range (620 - 300) [4].

2) The images were shown to 3 Radiologists trained and with wide experience in MR studies of the breast and according their opinion the phantom mimics the real human breasts.

\subsection{Performance of the Breast MRI Examinations}

At this point, the MRI examinations were started. These tests were conducted with a $1.5 \mathrm{~T}$ MRI system (Philips ${ }^{\circledR}$ ), using a bilateral breast surface coil with two sensitive areas. The examinations were performed such as the prone position.

The imaging protocol consisted of the pulse sequences shown in the Table 3 (number of slices = 50; Gap =0).

The study was started with the Survey in order to observe if the phantom was in isocenter and without any defects or needed corrections in the position. For the best
Table 3. Summary of acquisition parameters are presented the sequences STIR, SPIR and SPAIR and the variation in their parameters.

\begin{tabular}{ccccc}
\hline Sequence & $\mathrm{TR}^{*}(\mathrm{~ms})$ & $\mathrm{TE}^{* * *}(\mathrm{~ms})$ & $\begin{array}{c}\text { Frequency } \\
\text { offset }(\mathrm{Hz})\end{array}$ & $\mathrm{TI}^{* * * *}(\mathrm{~ms})$ \\
\hline STIR 1 & 8803 & 80 & - & 100 \\
STIR 2 & 8803 & 80 & - & 90 \\
STIR3 & 8803 & 80 & - & 135 \\
SPIR 1 & 3500 & 120 & 100 & - \\
SPIR 2 & 3500 & 120 & 80 & - \\
SPIR 3 & 3500 & 120 & 120 & - \\
SPAIR 1 & 3500 & 120 & 100 & - \\
SPAIR 2 & 3500 & 120 & 80 & - \\
SPAIR3 & 3500 & 120 & 120 & - \\
${ }^{*}$ TR (repetition time); ${ }^{* * *}$ TE (echo-time); ${ }^{* * *}$ TI (inversion time).
\end{tabular}

assessment of the images, the axial plane was chosen to be considered the best plane for the simultaneous evaluation of the two phantom breasts.

\subsection{Data Processing}

The data processing was performed on Image $J^{\circledR}$ software. Through this software after to build the structure of the visualization method, five clinical experts were asked to observe and to evaluate the MRI breast phantom images in a five-point qualitative Likert scale. These points were weighted towards increasing and being symmetric and balanced by having a central value and equal number of options laterally, 1 = very poor; 2 = poor; 3 = sufficient; 4 = good; 5 = excellent. The parameters under evaluation were:

1) Saturation uniformity;

2) Saturation efficiency;

3) Detail of the anatomical structures;

4) Differentiation of the fibroglandular tissue/adipose tissue.

For this purpose, it was performed a form titled "Perceptual Evaluation of Breast MRI Images” with the scale mentioned above, which the clinical experts fulfilled according to their opinions.

These experts are professionals from the staff of the Radiology department of an Oncology Institute. They were invited to collaborate in this study since all of them have over five years in experience on breast MRI examinations and gathered all the knowledge necessary for the intended evaluation. The group of experts was composed of three Radiologists and three Radiographers specialized in breast MRI.

From the amount of 300 images obtained, two images were selected from each sequence referred in the Table 3. 
The images selected were carefully chosen at the same spatial level of the breast, and the window was standardized to 277.5/482.4 values.

Statistical analysis was then performed on SPSS ${ }^{\circledR} 17.0$ software. The differences in the qualitative analysis were analyzed using the nonparametric Friedman Test.

\section{RESULTS}

The selected sample to perform the phantom was the most consistent with the proposed target, this mean, according to the breast anatomy.

The agarose and the gelatin (gelling agents) were used to provide the phantom sufficient strength and long enough $\mathrm{T} 1$ and $\mathrm{T} 2$ values to mimic the human breast tissue.

In order to simulate the heterogeneity of fat and fibroglandular tissue that occurs within breasts, the pork fat representing the breast adipose tissue and the turkey breast representing the breast parenchyma were put in different quantities on each breast. The left breast has less $20 \%$ of turkey breast and more $20 \%$ of pork fat than the right breast.

The Friedman test was applied to check if, according to the qualitative analysis of the experts, there are significant differences between the sequences for each parameter, individually. The Friedman test is a non-parametric statistical test, which through multiple test attempts is used to detect statistical differences.

The Friedman test is used when there are more than two terms of pairing, where each variable is classified in an ordinal scale.

As this study is based on the evaluation of four different parameters in nine sequences from five experts, this test was applied to determine if there is a difference between the sequences in the evaluation of the breast, according to the parameters.

The null hypothesis for this test is that there are no significant differences between the sequences.

This statistical method was applied to each parameter individually for each sequence [20].

The Fleiss' kappa coefficient revealed a good agreement among observers, $\mathrm{P}(e)=0.68$, where the evaluations obtained a mean value of 3.65 .

The sequences with worst assessment by the experts were SPIR 1 and SPIR 2 (Figure 6).

The obtained values by the Friedman test for the four parameters were $p>0.05$, which confirm the null hypothesis. In this case there are no statistically significances between the appliance of the STIR, SPIR or SPAIR to suppress the breast fat.

\section{DISCUSSION}

Among a variety of imaging methods used in the study

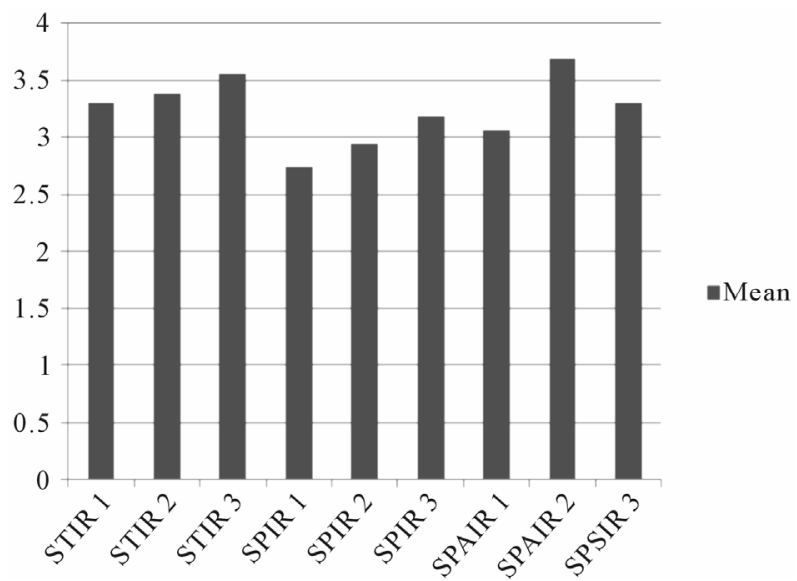

Figure 6. Mean values of the qualitative analysis of the experts.

of the breast, MRI becomes extremely important and indispensable. Contrast-enhanced MRI can demonstrate the tumour extent and multiplicity and is considered to be a powerful modality for detection and staging of breast cancer. The combined use of one efficacy fat suppression with enhanced MRI is essential [21].

Signal from fat plays a critical role in determining the useful contrast of images in many situations. Being able to control the signal developed by fatty tissue is a powerful and possibly an under-rated method in the MR imaging repertoire.

As showed in the Figure 6, the average of the analysis of the experts for each sequence has values very close to each other, which indicates that the expert's opinions converge to the same conclusion.

The disadvantages of fat suppression exist, such as increased magnetic susceptibility artifacts around air containing structures and lower signal-to-noise ratio; increase of the sequence time, decrease of uniformity between one similar structure. However, there are obvious advantages too, like the increased contrast between lesions and soft tissue compared with conventional MRI methods [10].

This study has shown some limitations. The fact that the breast phantom of this study didn't simulate any lesions could maybe contribute to a non-differentiated level of the pattern analysis. The fact that all experts demonstrated a high agreement was the strength of this study. This maybe has contributed to a similar response pattern, due to the similar professional experience of the experts-from the same imaging department and the similar mode to manage the breast MR images.

The lack of differences may occurred due the calibration procedures and check quality program over equipment and the optimization of the sequences carried out by the medical physics included on the department. This can promote stability on the homogeneity. The asset of 
this study was, in our opinion, the large number of MRI experiments. The problem was, that didn't allow the use of a human model due to the ethical procedures. Also there is still no study similar therefore we didn’t have a related article that could guide us.

\section{CONCLUSIONS}

Fat suppression is a generic term that includes various techniques, each with specific advantages, disadvantages and pitfalls.

The suppression of fat signal from breast tissue is often inconsistent and non-uniform. MRI Radiographers have access to a range of fat suppression methods, which work by exploiting particular characteristics of the MR (magnetic resonance) signals from fat and water. Understanding the mechanisms of each method provides insight into appropriate applications and situations where artifacts can be anticipated. Controlling the fat suppression can enhance the sensitivity and specificity of an MRI breast examination in many clinical settings [22], so the MR Radiographer must be familiar with the available fat suppression techniques and know when to apply them.

In this study, the obtained results by the Friedman Test, we observed, for the studied parameters, that there aren't significant advantages of one technique comparing to the others.

Each technique has anatomical, physiologic and technique justification for application and these characteristics depends sometimes of the morphometric, age and biotype of the patient.

It is recommended future studies development with optimization of the acquisition parameters in all MR departments and specific to each equipment in order to minimize some acquisition errors.

It is also proposed that the studies integrate different parameters and a panel of experts with a wide and different professional experience.

\section{ACKNOWLEDGEMENTS}

The authors thank to the Head of Radiology department of Oncologic Institute of Lisbon, and their Radiographers.

Also want to thank the volunteer to the mould, the Statistic, Prosthesis and Chemistry department's coordinators and their collaborators.

\section{REFERENCES}

[1] Hendrick, E. (2008) Breast MRI: Fundamentals and technical aspects. Springer, New York, 1-145. doi:10.1007/978-0-387-73507-8_1

[2] Medved, M., et al. (2006) High spectral and spatial resolution MRI of breast lesions: Preliminary clinical experience. American Journal of Radiology, 186, 30-37.
[3] Genson, C., et al. (2007) Effects on breast MRI of artifacts caused by metallic tissue marker clips. American Journal of Radiology, 188, 372-376.

[4] Ribeiro, M., et al. (2010) Técnicas de supressão de gordura: Estudo comparativo em ressonância magnética mamária. Acta Radiológica Portuguesa, XXII, 21-22.

[5] Schapiro, R., et al. (1986) Magnetic resonance imaging in the diagnosis of breast disease. American Journal of Radiology, 146, 119-125.

[6] Kopans, D. (1997) Magnetic resonance imaging. In: Ryan, J., Ed., Lippincott-Raven, 2nd Edition, Breast Imaging, New York, 617-634.

[7] American College of Radiology (2008) ACR practice guideline for the performance of contrast-enhanced MRI of the breast. Breast MRI, 25, 561-567.

[8] Mussurakis, S., Buckley, D., Drew, P., et al. (1997) Dynamic MR imaging of the breast combined with analysis of contrast agent kinetics in the differentiation of primary breast tumours. Clinical Radiology, 52, 516-526. doi:10.1016/S0009-9260(97)80328-5

[9] Delfaut, E., et al. (1999) Fat suppression in MR imaging: Techniques and pitfalls. Radiographics, 19, 373-382.

[10] Tien, R. (1992) Fat-suppression MR imaging in neuroradiology: Techniques and clinical application. American Journal of Radiology, 158, 369-379.

[11] Morrell, G.R. (2009) Rapid fat suppression in MRI of the breast with short binomial pulses. Journal of Magnetic Resonance Imaging, 24, 1172-1176. doi:10.1002/jmri.20737

[12] Riedl, E., et al. (2001) The role of STIR MRI sequence in the evaluation of the breast following conservative surgery and radiotherapy. Neoplasma, 48, 7-114.

[13] Lauenstein, T. (2008) Spectral adiabatic inversion recovery (SPAIR) MR imaging of the abdomen. Magnetom Flash, 2, 16-20.

[14] Kato, H., et al. (2005) Composition of MRI phantom equivalent to human tissues. The American Association of Physicists in Medicine, 32, 3199-3208.

[15] Boston, R., et al. (2005) Estimation of the content of fat and parenchyma in breast tissue using MRI T1 histograms and phantoms. Magnetic Resonance Imaging, 23, 591-599. doi:10.1016/j.mri.2005.02.006

[16] Madsen, E., et al. (2006) Anthropomorphic breast phantoms for qualification of investigators for ACRIN protocol 6666. Radiology, 239, 869-874. doi:10.1148/radiol.2393051070

[17] Greatrex, K., et al. (2005) Current role of magnetic resonance imaging in breast imaging: A primer for the primary care physician. The Journal of the American Board of Family Practice, 18, 478-490. doi:10.3122/jabfm.18.6.478

[18] Lee, N., et al. (1997) Fatty and fibroglandular tissue volumes in the breasts of women 20 - 83 years old: Comparison of X-ray mammography and computer-assisted MR imaging. American Journal of Radiology, 168, 501506.

[19] Pestana, M.H. and Gageiro, J. (2005) Análise de dados 
para ciências sociais-A complementaridade do SPSS. Edições Sílabo, Lisboa.

[20] Niitsu, M., et al. (2003) Fat suppression strategies in enhanced MR imaging of the breast: Comparison of SPIR and water excitation sequences. Journal of Magnetic Resonance Imaging, 18, 310-314. doi:10.1002/jmri.10364

[21] Lin, C., et al. (2010) Quantitative evaluation of fat suppression techniques for breast MRI at 3.0T. Proceedings of ISMRM Meeting 2010.

http://cds.ismrm.org/protected/10MProceedings/files/1_p rogram2.htm
[22] Brown, G. (1996) Fat suppression techniques. http://www.users.on.net/ spinupdownunder/papers/fatsup /fatweb.htm

[23] Lee, Y., et al. (2011) STIR versus SPAIR in breast imaging: A case-based discussion. Clinical Woman's Health. http://healthcare.siemens.com/siemens_hwem-hwem_ssx a websites-context-root/wcm/idc/siemens hwem-hwem ssxa websites-context-root/wcm/idc/groups/public/@glo bal/@imaging/@mri/documents/download/mdaw/mde2/ edisp/stir versus spair in breast imaging-00016900.pdf 